Pathologe 2008 • [Suppl 2] 29:383-387 DOI 10.1007/s00292-008-1086-z

Online publiziert: 28. September 2008

c) Springer Medizin Verlag 2008

H. Schäfer

Institut für Pathologie, Universitätsklinikum Hamburg-Eppendorf

\title{
Bericht über die Arbeitsgemeinschaft Paidopathologie
}

Aachen (B. Hermanns-Sachweh), Magdeburg (I. Röhse) und Hamburg (H. Schäfer; Schwerpunkt: kindliche Stoffwechselund Lebererkankungen) zu nennen. Aus dem deutschsprachigen Raum außerhalb Deutschlands haben sich im Rahmen der AG Paidopathologie u. a. Arbeitsgruppen aus Zürich, Aarau bzw. Chur (J. Briner, Th. Stallmach), Basel (E. Bruder), Innsbruck (C. Sergi), Wien (G. Amann) und Graz (M. Ratschek) besonders verdient gemacht.

Neben den jährlichen Sitzungen der AG Paidopathologie auf den Jahrestagungen der Deutschen Gesellschaft für Pathologie (DGP) treffen sich die an der Paidopathologie Interessierten seit $1988 \mathrm{zu}$ Vorträgen und informellem Erfahrungsaustausch bei den so genannen „Arbeitsgesprächen Deutschsprachiger Kinderpathologen“. In diesem Jahr hat unter Leitung von H. Schäfer die 16. dieser Tagungen am 26./27. Januar 2008 in Hamburg stattgefunden. Inhaltliche Hinweise auf einige der dabei gehaltenen Vorträge (die Abstracts wurden in der Zeitschrift Pediatric and Developmental $\mathrm{Pa}$ thology veröffentlicht) finden sich am Ende dieses Berichtes.

Die 17. Tagung der Deutschsprachigen Kinderpathologen wird noch in diesem Jahr unter Leitung von A. Müller am 14. bis 16 . November 2008 in Bonn stattfinden.

Im Folgenden seien einige Aspekte zur allgemeinen Situation der Paidopathologie in Deutschland erwähnt, die auf den beiden in diesem Jahr bereits stattgefundenen Treffen der Kinderpathologen (insbesondere auf der Tagung im Januar 2008) ausführlich diskutiert wurden.

\section{Perspektiven für eine zunehmende Relevanz der Paidopathologie}

1. Besonders in der Entwicklungs-, Stoffwechsel- und Tumorpathologie hat sich die diagnostische Treffsicherheit durch die Erkenntnisse der Molekularbiologie entscheidend verbessert. Trotzdem ist die Fähigkeit zur speziell qualifizierten deskriptiven Analyse paidopathologischer Fälle weiterhin unverzichtbar und durch Experten bewusst zu pflegen.

2. Weitere, die zunehmende Relevanz paidopathologischer Befunde unterstreichende Stichworte sind:

- autoptische Kontrolle klinisch erhobener pränataler Befunde (z. B. Ultraschall);

- genauere genetische Familienberatung durch differenziertere, die Molekularbiologie einbeziehende Diagnostik von Fehlbildungen und angeborenen Stoffwechselkrankheiten;

- differenziertere und sichere Diagnostik von Tumoren und Tumorsubentitäten mit der Möglichkeit einer differenzierteren prognostischen Aussage und Therapie;

- Etablierung und Indikationsstellung für spezifische Einzelfaktorgerichteter Therapiekonzepte bei Stoffwechselerkrankungen und Tumoren.

Anlässlich der Sitzung der Arbeitsgemeinschaft am 17. Mai 2008 auf der 92. Jahrestagung der Deutschen Gesellschaft für Pathologie (DGP), Berlin. 
3. Indikationsstellung und Kontrolle von Transplantations- und Stammzelltherapien im Kindesalter.

4. Spezielle Kenntnisse von embryonalen und fetalen Differenzierungsvorgängen sind wichtig auch für Stammzellkonzepte bei Erwachsenen.

5. Kontrolle der biologischen Relevanz von Ergebnissen der Grundlagenwissenschaften (z. B. In-vitro-Beobachtungen an Zellkulturen) durch Vergleich mit empirischen Befunden der Entwicklungspathologie.

\section{Probleme der Paidopathologie im deutschsprachigen Raum und mögliche Konsequenzen}

1. Bei Autopsien erfolgt, wie in anderen Bereichen der Pathologie, eine starke Zunahme der Verweigerungsraten. Dieses erfordert gemeinsam mit Klinikern und Genetikern eine intensivere Aufklärung der Angehörigen über die Relevanz der Autopsien (z. B. für die genetische Beratung).

2. Das Problem der inhaltlichen Überschneidung mit "Organpathologen" muss durch konstruktive Absprachen am Ort gelöst werden. Gefahren bestehen z. B. darin, dass den Kinderpathologen einerseits nur etattechnisch „unwirtschaftliche“ paidopathologische Problemfälle zugestanden und andernfalls wichtige Details von paidopathologisch nicht Versierten übergangen werden.

3. Eine adäquate paidopathologische Versorgung erfordert in den Versorgungsräumen die Verfügbarkeit entsprechender paidopathologischer Experten. Eine Durchführung paidopathologischer Autopsien nur durch „die jüngsten Assistenten“ ist obsolet.

4. Ein allgemein gültiges Rezept für die strukturelle Etablierung der Paidopathologie in den Instituten existiert nicht. Einerseits kann die breite paidopathologische Versorgung durch einzelne paidopathologische Experten (bei deren Abwesenheit mit Verfügbarkeit von entsprechenden Vertretern) gewährleistet werden. Andererseits muss unbedingt eine gewisse (noch zu diskutierende) Anzahl von längerfristig institutionalisierten, spe- zialisierten, in ihren Schwerpunkten untereinander abgestimmten Abteilungen für Paidopathologie existieren, die eine adäquate Ausbildung der disseminiert tätigen Paidopathologen gewährleisten und eine wissenschaftliche „Leuchtturmfunktion“ wahrnehmen. Freiraum von sonstigen Versorgungsaufgaben, notwendige Autonomie und Ressourcen dieser Abteilungen müssen gewährleistet sein.

5. Basiskenntnisse in der Genetik sind für den Paidopathologen unerlässlich. Das notwendige Ausmaß ist örtlich unterschiedlich und hängt von den jeweiligen Gegebenheiten in der Kooperation mit den Humangenetikern ab.

6. Wie in anderen Bereichen der Pathologie besteht auch in der Paidopathologie ein erheblicher Nachwuchsmangel. Um die Paidopathologie für den Nachwuchs attraktiv zu machen, sind u. a. folgende Voraussetzungen notwendig:

- Trotz Überscheidungen und Kooperationsbereitschaft müssen Profil und Zuständigkeitsbereiche der Paidopathologie klar definiert und erkennbar sein.

- Das Perspektiveangebot für den Nachwuchs muss verbessert werden.

- Überregional anerkannte Qualifikationsnachweise für Paidopathologen müssen geschaffen werden.

7. Für einen entsprechenden Qualifikationsnachweis wurden verschiedene Möglichkeiten (z. B. Zusatzbezeichnung „Kinderpathologie“, spezielle Qualifikationsbescheinigung der DGP u. a.) unter dem Gesichtspunkt der jeweiligen Vorteile und der Realisierbarkeit diskutiert. Ein hierfür zu erstellender Ausbildungskatalog sollte auch die Humangenetik und Molekularbiologie angemessen berücksichtigen.

8. Internationale Aktivitäten und Repräsentanz der deutschsprachigen Kinderpathologen in internationalen Fachgesellschaften („Paediatric Pathology Society“/PPS und „Society for Pediatric Pathology") sollten intensiviert werden.
Die begonnene Diskussion über die genannten Punkte wird auf den folgenden Sitzungen der deutschsprachigen Kinderpathologen fortgesetzt werden.

\section{Fachvorträge}

Die in der Sitzung der AG Paidopathologie auf der 92. Tagung der DGP am 15. Mai 2008 gehaltenen Fachvorträge behandelten ein breites Spektrum in der Fetal-, Fehlbildungs-, Tumor- und Stoffwechselpathologie. Folgendes sei berichtet:

In einem ausführlichen einleitenden Referat von K. Schoner (Marburg), H. Reh$\operatorname{der}$ (Wien) et al. wurde an 6 konkreten Beispielen mit verschiedenen Leitsymptomen (einmal mit Hydrops, 3-mal mit Polydaktylie, 2-mal mit Zwerchfelldefekt) das diagnostische Vorgehen bei der Syndromdiagnose in der Fetalpathologie demonstriert. Die Fälle belegten eindrucksvoll die Bedeutung einer möglichst genauen Syndromzuordnung u. a. auch für die genetische Familienberatung. Diese Zuordnung ist nur möglich auf der Basis einer insbesondere bei kleinen Feten oft sehr aufwendigen morphologischen Analyse der Fehlbildungen, die eine genaue Kenntnis der normalen Entwicklung voraussetzt. An den mit besonderer Expertise erhobenen und vorgestellten Befunden wurde auch die Unerlässlichkeit einer exakten molekularbiologischen bzw. zytogenetischen Bestandsaufnahme für eine sichere Zuordnung deutlich. Besonders eindrucksvoll war in der Polydaktyliegruppe die Darstellung des Smith-Opitz-LemliSyndroms, bei dem nicht nur die Diagnose durch eine akribische Analyse der Fehlbildungen gestellt werden konnte, sondern zugleich eine Mutation des Dehydro-Cholesterin-Rezeptor-7- (DHCR7-)Gens gefunden wurde. Da $\mathrm{DHCR}_{7}$ an der Testosteronbildung beteiligt ist, erklärte die gefundene Mutation, dass bei dem Syndrom solche Körperregionen (männliche Genitalorgane, Polydaktylie) fehlgebildet sind, deren Entwicklung einem Testosteroneinfluss unterliegt. Es gelang somit exemplarisch die Darstellung einer interessanten funktionellen Brücke zwischen Genmutation und Fehlbildungsmuster.

Die Autoren T. Braunschweig, J. Sachweh und B. Hermanns-Sachweh (Aachen) berichteten über ein Neugeborenes mit 
einer schweren Ateminsuffizienz, für die sich neben einer Fehleinmündung der Lungenvenen und einer Hypoplasie des Aortenbogens als ungewöhnliche Ursache eine schwere sekundäre Lymphangiektasie der Lunge herausstellte. Diese führte trotz operativer Korrektur der übrigen Gefäßanomalien zum Tode. Die vorliegende sekundäre Lymphangiektasie muss vom interstitiellen Emphysem und den ebenfalls seltenen primären pulmonalen Gefäßanomalien (z. B. Lymphangiose, Hämangiomatose, alveoläre kapilläre Dysplasie usw.) abgegrenzt werden, was teilweise den zusätzlichen Einsatz von Immunhistochemie (z. B. $\mathrm{CD}_{34}, \mathrm{D}_{2}-40$ ) und Elektronenmikroskopie erfordert.

Von M.S. Fahr, L. Seidmann, A.M. Müller, R. Schumacher und W. Coerdt (Mainz) wurde der zweite Fallbericht über ein neues kongenitales skelettales Syndrom mit Mikrozephalie und Rippenhypoplasie vorgestellt. Dieses hier zum ersten Mal bei einem männlichen Föten dokumentierte, autosomal-rezessiv erbliche Syndrom konnte durch die im Einzelnen geschilderte genaue Skelettanalyse von dem ähnlichen, aber häufigeren Seckel-Syndrom abgegrenzt werden, das klinisch zunächst vermutet worden war. Die postnatale Lebensfähigkeit ist bei dem vorliegenden Syndrom entscheidend eingeschränkt, sodass die Schwangerschaft in der 20. Schwangerschaftswoche unterbrochen wurde.

Die Autoren E. Bruder, A. PerezAtayde, G. Jundt, A.I. Alomari, J. Rischewski, S.J. Fishman, J.B. Mulliken und H.P.W. Kozakewich (Basel und Boston) gaben eine Übersicht über die insgesamt seltenen vaskulären Knochenläsionen bei Kindern, Adoleszenten und jungen Erwachsenen. Nach der Einteilung der „International Society for the Study of Vascular Anomalies“ (ISSVA) werden vaskuläre Malformationen von den verschiedenen Formen der proliferativen vaskulären Tumoren abgegrenzt. In dem vorliegenden, für die Diagnostik sehr relevanten Beitrag wurde der wichtige Versuch unternommen, die Brauchbarkeit und Anwendbarkeit der ISSVA-Klassifikation an einem bei der Seltenheit bemerkenswert großen Kollektiv von 77 vaskulären Knochenläsionen der oben angegebenen Altersklasse zu überprüfen. Dabei wurden auch röntgenologische und immunhistochemische Befunde (CD31, CD34, D2-40, GLUT1, MIB1) einbezogen. Die Ergebnisse belegen die Anwendbarkeit der ISSVA-Klassifikation und -Kriterien nicht nur für vaskuläre extraossäre, sondern auch für ossäre Läsionen. Sie ermöglicht (u. a. durch die GLUT1-Reaktion) eine klare Unterscheidung von Malformationen und Gefäßtumoren. Vaskuläre Malformationen waren häufiger als Gefäßtumoren bei etwa gleicher Häufigkeit von lymphatischen und venösen Malformationen. Bei den Tumoren waren Hämangioendotheliome und epitheloide Hämangiome besonders häufig. Die immunhistochemische Analyse eines bisher noch nicht beschriebenen Kaposi-formen Hämangioendotheliom-ähnlichen Tumors sprach für eine Interpretation dieses Tumors als Lymphangiomatose.

In einem Beitrag von T. Grob, R.Schmelzle und H. Schäfer (Hamburg) wurden an einem selbst beobachteten Fall aktuelle immunhistochemische und molekularbiologische Aspekte bei der bioptischen Diagnose des Cherubinismus präsentiert. Bei Kenntnis einer entsprechenden Familienanamnese und bei voller Ausbildung der typischen Gesichtsdeformitäten ist die Diagnose unproblematisch. In der noch uncharakteristischen Frühphase können die Diagnose und die Unterscheidung von anderen fibroblastischen riesenzellhaltigen osteolytischen Läsionen des Kiefers jedoch schwierig sein. An dem präsentierten, bioptisch im Verlauf vorliegenden Fall wurden die histologischen Veränderungen und die immunhistochemische Abgrenzung differenzialdiagnostisch infrage kommender Läsionen (z. B. der zentralen Riesenzellläsion des Kiefers, der Langerhans-ZellHistiozytose usw.) dargestellt. Pathognomonisch aber war der molekularbiologische Nachweis einer typischen Mutation des $\mathrm{SH}_{3} \mathrm{BP}_{2}$-Gens in Position 4p16.3, das ein c-Abl-bindendes Protein kodiert. Praktisch wichtig ist auch, dass diese $\mathrm{Mu}$ tation als Keimbahnmutation schon in der Frühphase des Cherubinismus nachweisbar ist und nicht nur eine Unterscheidung von den anderen genannten Tumoren, sondern auch eine Abgrenzung vom Noonan-Syndrom erlaubt, bei dem eine Mutation des PTN11-Gens in Position 12q.24.1 vorliegt, dessen riesenzellhaltige
Läsionen ähnlich sind, das sich aber durch assoziierte Herzfehlbildungen vom Cherubinismus unterscheidet.

Von A.Poeschl, T.Branschweig, J.Senderek und B. Hermanns-Sachweh (Aachen) wurde der genetische Hintergrund der kongenitalen A-bzw. Hypoplasie der Fibula (FAH) dargestellt. Diese häufigste Form der longitudinalen Skeletterkrankungen ähnelt morphologisch einem bestimmten Knock-out-Modell der Maus mit einem Defekt für das Homeobox-Gen hox c11. In der vorliegenden Studie wurde erstmalig an 14 Patienten geprüft, ob auch bei der FAH des Menschen ein Defekt des hox 110 oder 11 vorliegt. Dieses wurde nicht gefunden. Die Untersuchungen sollen auf weitere Kandidatengene (Hox d13, Shh und Wnt7a) ausgedehnt werden.

C. Falkeis, B. Brunner, G. Mikuz, E. Streichen und C. Sergi (Innsbruck) berichteten über einen selbst beobachteten Fall mit Perlman-Syndrom, das durch eine typische Gesichtsform, fetalen Gigantismus, Viszeromegalie und renale Hamartome mit oder ohne Nephroblastomatose bzw. Wilms-Tumor charakterisiert ist. Besonders herausgestellt wurden die differenzialdiagnostisch wichtigen Ähnlichkeiten und Unterschiede dieses autosomal-rezessiv erblichen Syndroms zum Beckwith-Wiedemann- und Simpson-Golabi-Syndrom, wobei die Einbeziehung bestimmter, im Einzelnen dargestellter histologischer und genetischer Kriterien essenziell ist.

H. Schäfer (Hamburg) gab eine ausführliche Übersicht über Krankheitsbeispiele, an denen die trotz Immunhistochemie und Molekularbiologie persistierende Bedeutung der Elektronenmikroskopie in der paidopathologischen Diagnostik erkennbar ist. Wohlbekanntes Bespiel hierfür ist die Identifikation bestimmter glomerulärer Basalmembranerkrankungen (z. B. „dünne Basalmembran“Krankheit, M. Alport/hereditäre Nephritis, „Minimal-change-Glomerulopathie“). Ein weiteres Beispiel ist die primäre Identifikation unvermuteter Virusinfektionen bei klinisch unklaren Fällen sowie von bestimmten Speicherkrankheiten mit pathognomonischen ultrastrukturellen Kriterien (z. B. M. Niemann-Pick/ Differenzialdiagnose M. Gaucher, M. Fabry, einer spezielle Fibrinogenspeicher- 
krankheit der Leber usw.). Bei den kongenitalen Mitochondriopathien (z. B. bei primären Atmungskettendefekten) kann über die grundsätzliche Diagnose hinaus die Anzahl der pro Zelle betroffenen, mosaikartig verteilten Mitochondrien und damit die biologische Relevanz im Einzelfall elektronenmikroskopisch abgeschätzt werden. Bei bestimmten kongenitalen Bürstensaumerkrankungen (z. B. Ziliendyskinesien, kongenitale Mikrovillusatrophie) ist die elektronenmikroskopische Diagnostik essenziell, da sonstige allein beweisende Kriterien für diese klinisch meist sehr gravierenden Erkrankungen fehlen. Die elektronenmikroskopische Diagnostik erfordert spezielle Expertise und ist sehr aufwendig; ihr Betrieb an bestimmten Zentren ist aber für die paidopathologische Diagnostik als Ergänzung der immunhistochemischen und molekularbiologischen Befunde weiterhin unerlässlich.

Von M.S. Fahr, L. Seidmann, U. Zeigmeister, M. Lockets und W. Coerdt (Mainz) wurde der interessante Fall eines Foeten vorgestellt, bei dem Hinweise auf einen bisher wenig bekannten Zusammenhang zwischen gesteigerter maternaler Manganaufnahme und fetalen Fehlbildungen bestehen. Bei der Mutter konnte ein langfristiger Gebrauch von Trinkwasser mit erhöhtem Mangangehalt gesichert werden. Der Foetus zeigte Extremitätenfehlbildungen mit dem Aspekt einer Arthrogryposis multiplex. Histologisch ergab sich außerdem der überraschende Befund von multiplen Verkalkungen $u$. a. in der Leber und in den Meningen der Wirbelsäule. Eine rasterelektronenmikroskopische Untersuchung der Verkalkungen unter Verwendung der energiedispersiven Röntgenmikroanalyse ergab allerdings nur den Nachweis von Kalzium, nicht aber von Mangan. Trotzdem liegt eine Beziehung zwischen der gesteigerten Manganexposition der Mutter und den fetalen Veränderungen nahe. Bei dem von Selye beschriebenen tierexperimentellen Modell der Kalziphylaxie kann eine Metallsalzapplikation eine schlagartig einsetzende Verkalkung im Gewebe bahnen, wobei sich dann sukzessive die applizierten Metallsalze durch endogen präzipitierende Kalziumsalze austauschen, was den negativen Manganbefund bei der Ge- websanalyse erklären könnte. Die Autoren regen an, bei der Erklärung fetaler Anomalien auch auf die Möglichkeit einer gesteigerten Metallexposition zu achten.

Der auf den reichen Erfahrungen des Kindertumorregisters basierende Vortrag von C. Vokuhl, J. Stöfen, S. Stegmaier, E. Koscielniak und I. Leuschner (Kiel und Stuttgart) befasste sich mit dem synovialen Sarkom im Kindes- und Adoleszentenalter. An einem großen Kollektiv von 63 synovialen Sarkomen im Alter bis zu 18 Jahren sollte geprüft werden, ob die in der Literatur allgemein für das synoviale Sarkom angegebenen ungünstigen Prognosekriterien bezüglich der Überlebensraten auch bei der untersuchten Altersklasse gelten. Als potenzielle Prognoseparameter geprüft wurden der histologische Subtyp, die mit verschiedenen immunchemischen Parametern evaluierte proliferative Aktivität, die immunhistochemische p53-Expression und der durch RT-PCR ermittelte Gen-Fusions-Typ. Im Gegensatz zu den beim synovialen Sarkom allgemein publizierten Ergebnissen anderer Arbeitsgruppen konnte bei dem vorliegenden pädiatrischen Kollektiv keine Beziehung der untersuchten Parameter zur Überlebensrate gefunden werden. Dieser Unterschied in der Relevanz der Prognoseparameter wird von den Autoren dahingehend interpretiert, dass es sich bei den synovialen Sarkomen im Kindesbzw. Jugendalter und im Erwachsenenalter möglicherweise um unterschiedliche Tumorentitäten handelt.

Die Autoren A. Zimpfer, D. Kaayser, P. Schwabbauer, H.-J. Feickert, I. Leuschner und E.W. Herbst (Neubrandenburg und Kiel) präsentierten einen Speicheldrüsenanlagentumor (kongenitales pleomorphes Adenom) des Nasopharynx als seltene Ursache einer neonatalen Atemwegsobstruktion. Dieser Tumor ist mit bisher nur 25 publizierten Fällen selten. Histologie und immunhistochemische Befunde dieses meist bei männlichen Patienten auftretenden Tumors entsprechen dem üblichen pleomorphen Adenom. Der Tumor ist benigne und durch einfache Resektion zu behandeln.

In einem die Sitzung abschließenden Vortrag stellten A.M. Müller, M. Kaucevic, W. Coerdt, M. Heinz und S. Turial (Mainz) die Frage, wieso sich so viele chro- nisch bzw. gar nicht entzündete Appendizes im täglichen paidopathologischen Einsendegut befinden. In dem großen untersuchten Kollektiv von 872 Appendektomiefällen im Alter zwischen 55 Monaten und 15 Jahren fand sich in nur $48 \%$ eine akute Appendizitis und nur in 52\% lediglich eine chronische oder fehlende Entzündung. Bei beiden Gruppen wurden einige typische klinische Symptome, Untersuchungsbefunde und Laborwerte verglichen. Leukozytose und CRP-Wert waren bei den Fällen mit akuter Appendizitis gegenüber der Gruppe mit chronischer bzw. fehlender Appendizitis zwar deutlich erhöht. Die übrigen detailliert im Einzelnen registrierten klinischen Veränderungen (z. B. Druck- und Loslassschmerz, Übelkeit, Erbrechen, freie Flüssigkeit in der Bauchhöhe usw.) fanden sich aber auch bei einem relevanten Anteil von $\mathrm{Pa}$ tienten mit chronischer bzw. fehlender Appendizitis. Dieses Fehlen sicherer Ausschlusskriterien einer akuten Appendizitis erklärt die hohe Rate von vorsorglich vorgenommenen Appendektomien, bei denen sich nachträglich der Verdacht auf eine akute Appendizitis nicht bestätigte.

\section{Tagung Deutschsprachiger Kinderpathologen}

Die Aktivität der AG Paidopathologie im laufenden Jahr wurde auch an dem reichhaltigen Vortragsprogramm auf der von H. Schäfer am 26./27. Januar 2008 in Hamburg organisierten „16. Tagung Deutschsprachiger Kinderpathologen" deutlich. Die dort präsentierten Vorträge seien im Folgenden nur thematisch erwähnt. Bezüglich Einzelheiten wird auf die in der internationalen Zeitschrift Pediatric and Developmental Pathology veröffentlichten Abstracts verwiesen.

\section{Sitzung über Tumoren}

D. Harms (Kiel) hielt ein ausführliches und instruktives Übersichtsreferat über „Histiozytäre Tumoren im Kindesalter“. Klinische und molekularbiologische Aspekte histiozytärer Erkrankung, die z. B. beim M. Farqhar erstmalig auch eine molekularbiologische Diagnostik ermöglichen, wurden von G. Janka-Schaub (Hamburg) vorgetragen. K. Ernestus, 
I. Leuschner et al. (Kiel und Köln) berichteten über die Prognose und Inzidenz des undifferenzierten und gering differenzierten Neuroblastoms nach der INPCKlassifikation. Der Beitrag von A. Quaas, U. Reichelt, H. Schäfer et al. (Hamburg) behandelte die Immunhistochemie einiger potenziell therapeutisch relevanter Marker (z. B. c-kit, EGFR, Her2-neu) beim Neuroblastom. Der Neuropathologe H. Hagel und der Pädiater U. Kordes (Hamburg) referierten gemeinsam über neue Aspekte der (u. a. molekularbiologischen) Diagnostik und Therapie kindlicher Hirntumoren (Astrozytome, Medulloblastome, atypische teratoide Rhabdoidtumoren). C. Vokuhl, I. Leuschner et al. (Kiel) berichteten über die Synovialsarkome im Kindesalter. E. Bruder, E. Jundt et al. (Basel) stellten einen multifokalen vaskulären Spindelzelltumor mit Skelettbeteiligung und Koagulopathie als mögliche eigene Entität vor. T. Wagner und A. Gnekow (Augsburg) schilderten den Überraschungsbefund einer Aktinomykose bei einem Mädchen mit klinischem Verdacht auf einen malignen Thoraxwandtumor. Von K. Wieczorek (Dresden) wurde der seltene Fall eines nasalen mesenchymalen Hamartoms vorgestellt.

\section{Sitzung über Entwicklungsstörungen}

Hier berichteten E. Hilbert, A. Schneider, W. Coerdt und A.M. Müller (Mainz) über eine im Gegensatz zur Präeklampsie bei Raucherinnen gesteigerte Expression des nikotinischen Acetylcholinrezeptors alpha 7 in der Plazenta. E.F. Karpf, B. Poetsch et al. (Graz) demonstrierten einen endometrialen Stromaknoten in einer Plazenta am Termin. N. Sarioglu und $W$. Henrich (Berlin) gaben eine reich illustrierte Übersicht über die klinische, ultrasonographische und morphologische Diagnostik der monochorialen Zwillingsschwangerschaft. M.S. Fahr (Mainz) präsentierte die Kasuistik einer kongenitalen azinären Dysplasie bei einem Foeten der 23. Schwangerschaftswoche. K. Schoner und H. Rehder (Marburg) stellten einen Fall vor mit der schweren Manifestation einer akrofazialen Dysostose mit Gesichtsspalten als mögliche Folge einer mütterlichen Immunsuppression mit Myco- phenolatmofetil in der Schwangerschaft. T. Braunschweig und B. Hermanns-Sachweh (Aachen) sprachen über die „Shortrib-Syndrome“ am Beispiel des JeuneSyndroms. Der Vortrag von B. HermannSachweh, T. Braunschweig et al. (Aachen) behandelte das Meckel-Gruber-Syndrom in differenzialdiagnostischer Abgrenzung zu den so genannten „Joubert-Syndrom related Disorders".

\section{Vortragsblock über Leber- und Stoffwechselerkrankungen}

In diesem hielt zunächst C. Sergi (Innsbruck) ein Referat über Cholestaseerkrankungen im Neugeborenenalter, unter besonderer Berücksichtigung von Entwicklungsstörungen der Portalfelder und der Duktalplatte. Es folgte ein Referat von R. Kubitz et al. (Düsseldorf) über hepatobiliäre Transportproteine bei cholestatischen Syndromen, das auch die Möglichkeiten der immunhistochemischen Erfassung bestimmter Transport-Protein-Defekte bei der progressiven familiären intrahepatische Cholestase (PFIC) darstellte. Das anschließende Referat von R. Santer, K. Tsiakas, H. Schäfer et al. (Hamburg) stellte die verschiedenen Formen der mitochondrialen DNA-Depletionssyndrome als Ursache von Leberkrankheiten bei Kindern dar, unter besonderer Berücksichtigung neuer klinischer, molekularbiologischer und histologischer Gesichtspunkte.

\section{Varia}

Im Abschnitt Varia berichteten A. Block, H. Wulff, H. Schäfer et al. (Hamburg) über die Entwicklung ultrahocheffizienter und regulierbarer adenoviraler Expressionssysteme und deren mögliche therapeutische Option für maligne sowie metabolische pädiatrische Erkrankungen. $\mathrm{K} . \mathrm{Be}$ cker, J. Bohl et al. (Kaiserslautern) präsentierten einen ungewöhnlichen Fall von plötzlichem Herztod im Kindesalter, der durch eine spezielle nichtobstruktive hypertrophische Kardiomyopathie bei gleichzeitig bestehender makrophagischer Myofasziitis mit (möglicherweise Impfstoff-induzierten) AluminiumhydroxydAblagerungen bedingt war. M. Kaucevic, W. Coerdt, A.M. Müller et al. (Mainz) be- handelten in ihrem Vortrag die Frage, welche klinischen Parameter im Kindes- und Jugendalter prädiktiv für eine pathomorphologisch akut entzündete Appendix sind. J. Matschke (Hamburg) präsentierte eigene Untersuchung aus dem pathologischen und rechtsmedizinischen $\mathrm{Ob}$ duktionsgut zu Ursachen, Häufigkeit und forensischer Relevanz von Subduralblutungen im Säuglingsalter. Den ermutigenden Abschluss der Tagung bildete der Vortrag des Rechtsmediziners J. Sperhake (Hamburg) über den plötzlichen Säuglingstod (SIDS) in Hamburg - Geschichte einer erfolgreichen Prävention.

Zusammenfassend belegen die geschilderten Vortragsprogramme auf den beiden bisher in diesem Jahr stattgefundenen Tagungen eine erfreuliche Aktivität der AG Paidopathologie. Die 17. Tagung der Deutschsprachigen Kinderpathologen wird - von A. Müller organisiert - bereits am Ende dieses Jahres vom 14. bis 16. November 2008 in Bonn stattfinden.

\section{Korrespondenzadresse}

Prof. Dr. H. Schäfer

Institut für Pathologie, Universitätsklinkum Hamburg-Eppendorf

Martinistr. 52, 20246 Hamburg

schaefer@uke.uni-hamburg.de

Interessenkonflikt. Der korrespondierende Autor gibt an, dass kein Interessenkonflikt besteht. 